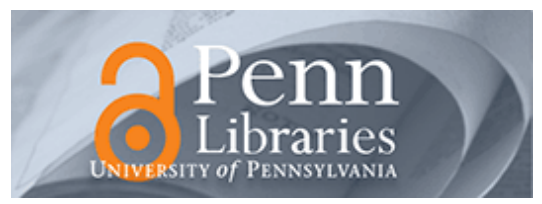

Academic Entrepreneurship for Medical and Health Scientists

Volume 1

Issue 3 Intellectual Property-Regulatory

Article 16

$10-9-2019$

\title{
Overview of Drug Development
}

Nalaka Gooneratne

Perelman School of Medicine, University of Pennsylvania

Follow this and additional works at: https://repository.upenn.edu/ace

Part of the Entrepreneurial and Small Business Operations Commons

\section{Recommended Citation}

Gooneratne, Nalaka (2019) "Overview of Drug Development," Academic Entrepreneurship for Medical and Health Scientists: Vol. 1: Iss. 3, Article 16.

Available at: https://repository.upenn.edu/ace/vol1/iss3/16

This paper is posted at ScholarlyCommons. https://repository.upenn.edu/ace/vol1/iss3/16

For more information, please contact repository@pobox.upenn.edu. 
The Academic Entrepreneurship for Medical and Health Scientists book project is free to all - we don't ask for money but we truly value your feedback.

Below are two links -- one to a brief feedback survey and the other to a place where you can sign up to join our community of innovators and problem solvers. You can visit them and give tell us what you think now OR after you've had the chance to read this chapter -- either one works for us!

Please complete our brief feedback survey https://redcap.chop.edu/surveys/?s=HDXK3CE48L

Join our growing community of Academic Entrepreneurs! https://bit.ly/3bnWTuD

\section{Overview of Drug Development}

\section{Summary}

For latest version: please go to https://academicentrepreneurship.pubpub.org/pub/8a05fz36/release/4

- Drug development costs range from $\$ 2$ to $\$ 3$ billion and may span from 7 to 12 years.

- Beginning with drug discovery, key steps in the process include preclinical testing, Phase 1-3 clinical trials, FDA New Drug Application (NDA) approval, and subsequent Phase 4 clinical research.

- Academic entrepreneurs should understand the unique needs of each phase and their potential role in the process, as well as the services their academic medical center can provide.

\section{Creative Commons License}

\section{(c) 1 (1)}

This work is licensed under a Creative Commons Attribution-Noncommercial-No Derivative Works 4.0 License. 


\title{
Overview of Drug Development
}

\author{
Nalaka Gooneratne, MD, MS ${ }^{1}$
}

Idea

Exit

Topic Relevance by Timeline

\section{Summary}

- Drug development costs range from $\$ 2$ to $\$ 3$ billion and may span from 7 to 12 years.

- Beginning with drug discovery, key steps in the process include preclinical testing, Phase 1-3 clinical trials, FDA New Drug Application (NDA) approval, and subsequent Phase 4 clinical research.

- Academic entrepreneurs should understand the unique needs of each phase and their potential role in the process, as well as the services their academic medical center can provide.

\section{Introduction}

Drug development is one of several cornerstones of advancing medical care. The process is complicated, expensive, and can span over a decade. An academic entrepreneur has several crucial roles to play throughout the process, and the resources available at academic centers - such as NIH-funded Clinical and Translational Science Awards (CTSA) and the tech transfer office-can often significantly contribute as well. In this chapter, we will provide an overview of the drug development process and the steps required to successfully bring a new compound from discovery to clinical practice. Other chapters provide information about specific subtypes of compounds, such as orphan drugs ("Orphan Drugs: Understanding the FDA Approval Process"), biologics ("Intellectual Property Protection for Biologics"), or radiographic agents ("Development of Radiographic Contrast Agents for Diagnostic Imaging”).

\footnotetext{
${ }^{1}$ Perelman School of Medicine, University of Pennsylvania
} 


\section{Background}

The Food and Drug Administration (FDA) approves approximately twenty-fifty new compounds each year, with 2018 seeing fifty-nine approvals (Figure 1), an unusually high number (Mullard). However, these simple numbers belie an important fact: drug approval is a costly and uncertain endeavor. The average cost of a new drug approval is $\$ 2-\$ 3$ billion, and only $14 \%$ of drugs that enter clinical trials eventually obtain FDA approval (DiMasi et al.; Wong et al.). This journey, from invention to clinical application in patients, can take from 7 to 12 years for a drug, compared to three to seven years for a device (Van Norman). Despite these daunting prospects, the task of drug development is crucial for advancing healthcare. New medications, along with several other key innovations in public health and other domains, have meaningfully contributed to the substantial increases in quality of life, functional status, and lifespan that have occurred over the past century.

Figure 1. FDA Drug Approvals per Year.

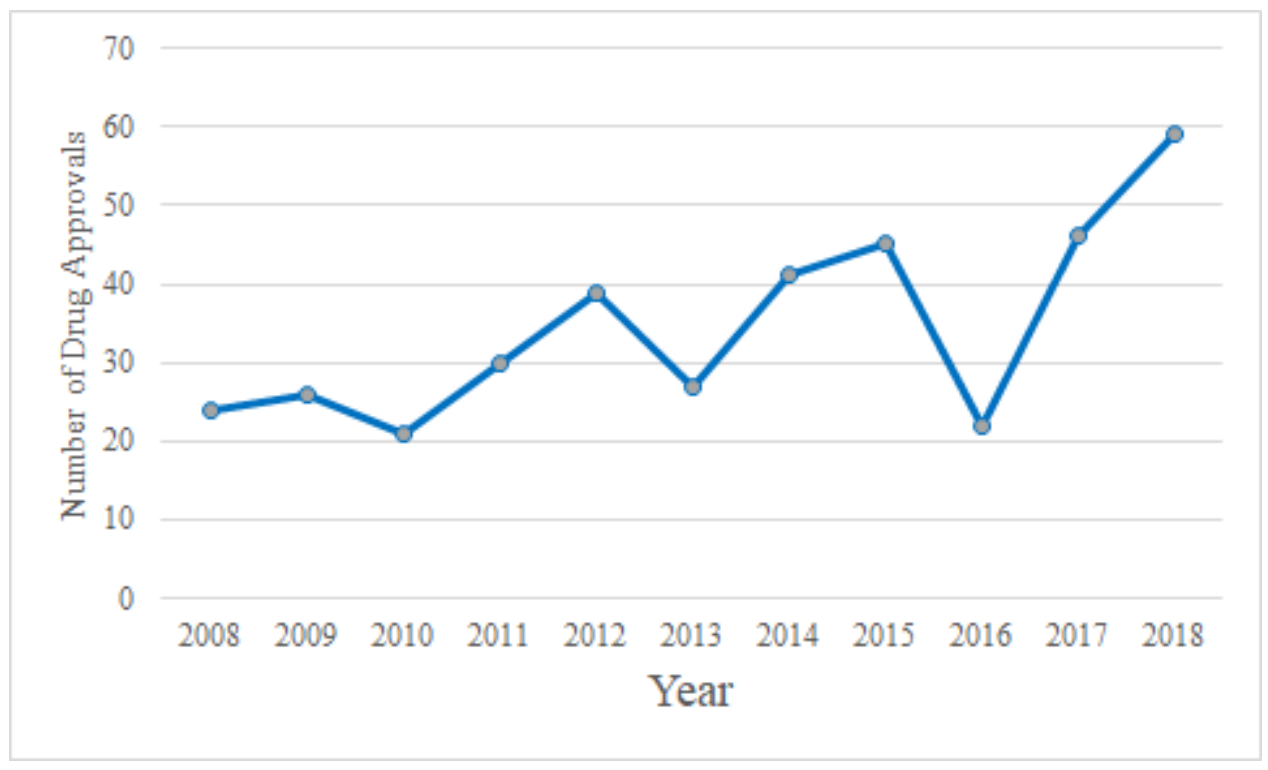

Source: Adapted from Mullard. Does not include vaccines.

\section{Discovery}

Drug discovery can occur through different pathways. These include 1) repurposing an existing molecule; 2) creating variants of an existing molecule (such as through cis/trans isomers); 3 ) identifying a molecule that acts on a specific biochemical pathway (perhaps one that the academic entrepreneur has been researching), or 4) finding a new compound in nature that can be used in humans or animals. Once identified, these compounds typically undergo a rigorous drug design process, as shown in Figure 2. Subsequently, they can be produced under Current Good Manufacturing Practice (CGMP) regulations, which are enforced by the FDA, culminating in an 
optimized candidate compound for subsequent evaluation in further preclinical (i.e., animal) and human studies.

Figure 2. Drug Design.

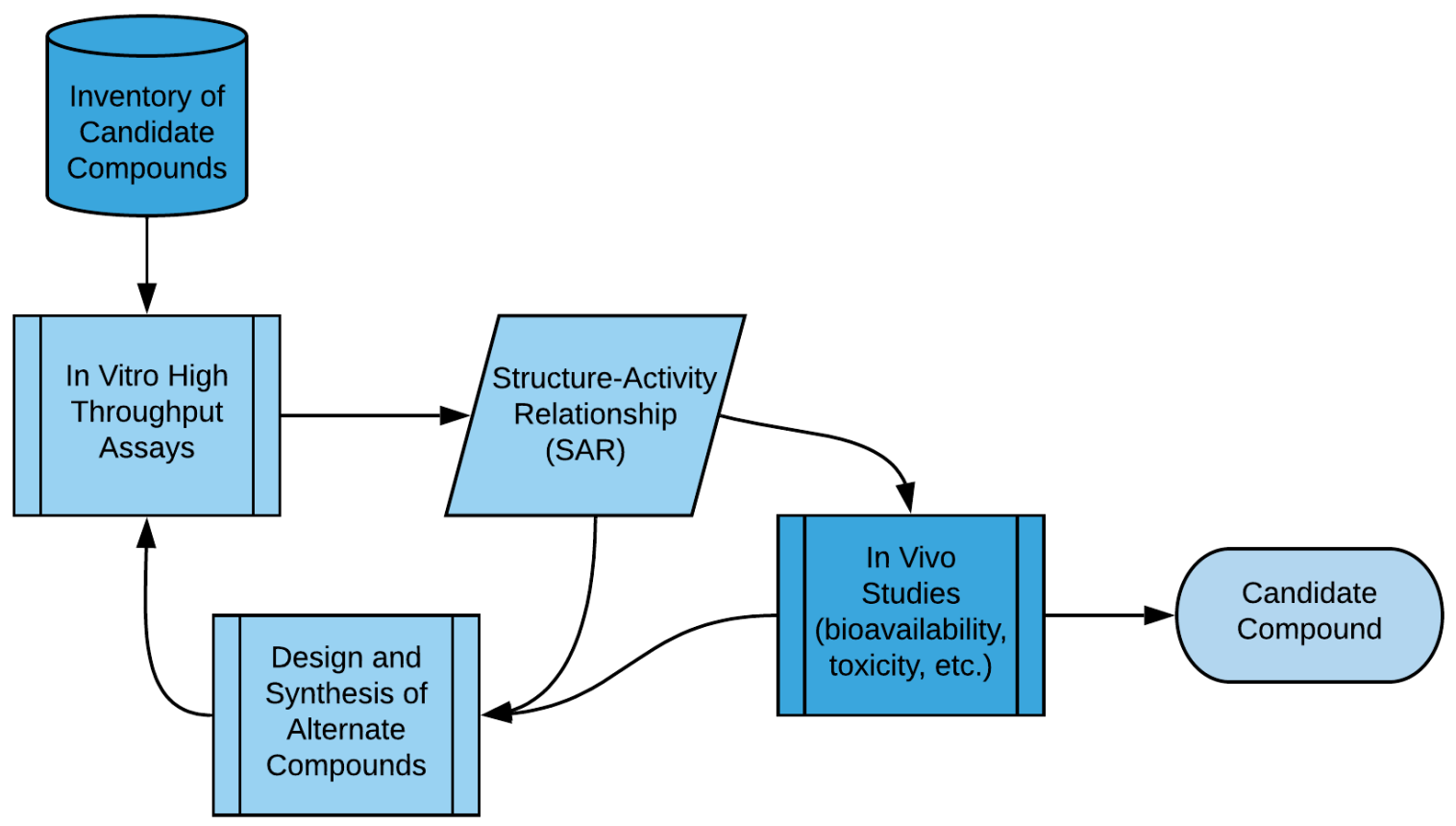

Source: Adapted from “Drug Design,” Wikipedia (Wikipedia contributors).

After the initial discovery phase, the compound may move on to additional FDA-required preclinical testing. Note that if an academic entrepreneur has the potential to benefit financially from the compound, either through licensing revenue or equity in a company that owns the compound or plans to develop it, some universities may require that the academic entrepreneur modify their role in regards to any research that may subsequently be included in FDA documents, starting with preclinical studies and extending through all phases of development (see the chapter "Understanding Conflict of Interest for Academic Entrepreneurs"). This may include relinquishing the principal investigator role and hiring an independent party to conduct the relevant research or audit the work. The first step in determining the appropriate measures is to contact the university's conflict-ofinterest committee prior to conducting any preclinical or clinical research that may be included in an FDA submission; this can avoid the costly outcome of repeating the work and subsequent delays in the FDA Investigational New Drug (IND) submission.

Since the steps below are primarily targeted toward obtaining FDA approval, it is crucial to engage with the FDA throughout the drug development process to make sure that the research studies are collecting the necessary data, and that there are no unanticipated surprises in terms of the need to conduct additional research studies (Figure 3). 
Figure 3. FDA Interactions During Drug Development.

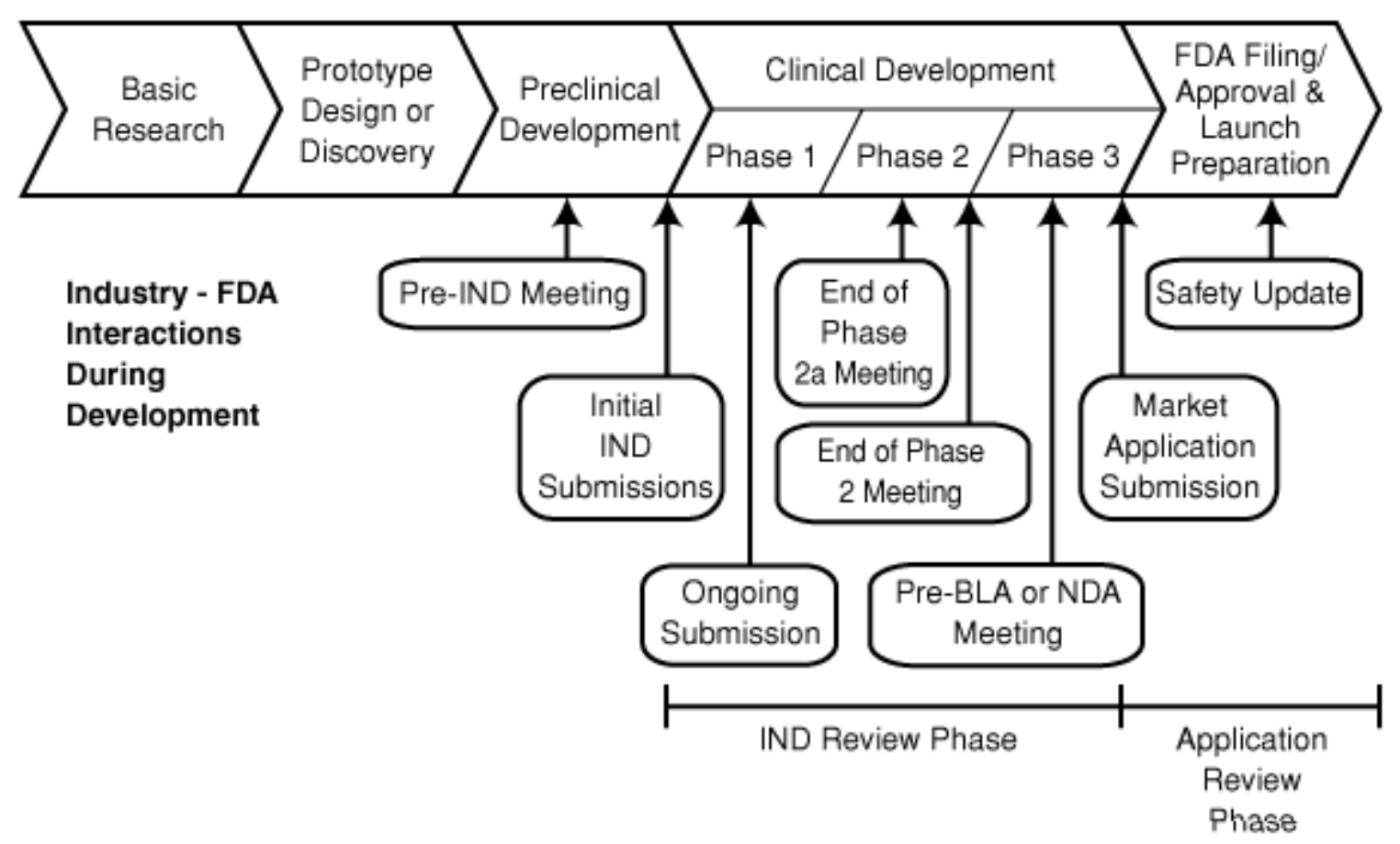

Source: Image downloaded from Wikimedia Commons and provided by the FDA:

https://commons.wikimedia.org/wiki/File:The_extensive_industry-

FDA_interactions that_occur_during product_development, using the_drug development_pro cess_as_a_specific_example.gif.

One particularly useful tool for conducting this engagement and ensuring that the subsequent development process is timely and efficient is a Target Product Profile (TPP). As described by the FDA, the goal of a "TPP is to provide a format for discussions between a sponsor and the FDA that can be used throughout the drug development process, from pre-investigational new drug application (pre-IND) or investigational new drug application (IND) phases of drug development through postmarketing programs to pursue new indications or other substantial changes in labeling. The TPP embodies the notion of beginning with the goal in mind. That is, the sponsor specifies the labeling concepts that are the goals of the drug development program, documents the specific studies intended to support the labeling concepts, and then uses the TPP to assist in a constructive dialogue with the FDA. The ideal version of what the sponsor would like to claim in labeling guides the design, conduct, and analysis of clinical trials to maximize the efficiency of the development program" (Guidance for Industry and Review Staff Target Product Profile-A Strategic Development Process Tool). 


\section{Preclinical Studies}

Preclinical studies are in vivo studies that usually occur in animals. Initially, they are conducted in animals without the disease of interest in order to test toxicology, then later in animal models of the disease that allow for assessment of disease-modifying effects. The purpose of this research is to obtain FDA approval for an IND that will in turn allow for subsequent research in human subjects. Testing must typically occur in two different mammalian species, as per FDA guidelines. The tests are often conducted by a center with expertise in this area; academic centers with veterinary schools may offer this service, as well as specialized outside companies (see the chapter "Preclinical Animal Models").

Note that the core preclinical studies must be completed before the compound is given to any human subjects. These studies can help determine the no-observed-adverse-effect levels (NOAELs) for the compound, which are necessary to identify the initial dose level of the compound for subsequent Phase 1 human clinical trials (first-in-man). Failure to follow FDA guidelines for conducting preclinical evaluations may result in an IND application being rejected, and culminate in the need to repeat the testing, potentially leading to significant delays in drug development. These FDA guidelines include rigorous quality control, oversight, and comprehensive reporting of results. This can be particularly challenging for academic entrepreneurs because, in some cases, this initial work would have been done in an exploratory capacity and thus may not meet FDA guidelines. On average, the preclinical phase can take three and a half years ("Drug Approvals - From Invention to Market...12 Years!’).

It is also worth noting that these preclinical studies may suggest a potential role for the compound in animal diseases and thus a veterinary medicine application for the compound. This offers several important benefits, including the potential to gather additional safety data and faster access to the veterinary market as compared to human applications, which can lead to revenue from sales in the veterinary medicine market that may support further development in humans. These opportunities are described in more detail in the chapter "Development Strategies for Animal Medical Therapeutics."

\section{Investigational New Drug Application}

Once the compound can be manufactured with optimal quality control, and once a sufficient body of evidence is accumulated from preclinical testing, the pharmaceutical company will next move to filing an Investigational New Drug (IND) application with the FDA. The purpose of an IND is to allow the FDA to evaluate the compound and determine if it is safe to begin testing the compound in humans. The FDA has 30 days to review the IND, and may request additional information that may require additional preclinical research and substantially delay approval of the IND, thus early engagement with the FDA to map out or review the preclinical testing prior to submitting the IND is highly recommended. The IND also includes detailed information about the planned human 
subject studies (Phases 1-3), and is often also reviewed by the institutional review board (IRB), which will be overseeing those studies. Additional details regarding the IND application are provided in the chapter "FDA Drug Regulation: Investigational New Drug Applications."

\section{Phase 1 Clinical Research}

After an IND has been approved by the FDA, or 30 days have elapsed since submission to the FDA without comment, the pharmaceutical company may begin testing in human subjects in Phase 1 trials. These studies typically involve 20 to 60 healthy volunteer participants. Starting with the NOAEL, adjusting for a safety factor, small groups are tested at sequentially higher doses to determine the safe dosage range. Other key parameters include pharmacokinetic characteristics such as bioavailability, distribution, and half-life. Academic research centers often have clinical trial units, in some cases funded by their Clinical and Translational Science Award (CTSA), which can be ideal venues for conducting this type of research because they are staffed by research nurses with experience in conducting early-stage human research studies (see the chapter "Clinical and Translational Science Awards"). Private, for-profit clinical research organizations often conduct these studies as well. Phase 1 trials may take an average of one year and are seminal for deciding whether or not to proceed with drug development ("Drug Approvals - From Invention to Market...12 Years!").

\section{Phase 2 Clinical Research}

Phase 2 clinical studies differ from Phase 1 in that the former involve patients with the disease of interest. They tend to be larger, involving up to 300 patients, and may be randomized. In some cases, they can be conducted at a single research site, but may often be multisite studies. A key goal is to determine the minimum and maximum dose of the compound, as well as potential effectiveness. In some cases, especially with cancer therapeutics, a drug may have very limited Phase 1 testing and may move quickly to Phase 2 trials. Phase 2 typically takes about two years ("Drug Approvals - From Invention to Market...12 Years!”).

As with Phase 1 studies, Phase 2 studies can also be conducted in for-profit contract research organizations (CROs) and academic medical centers; CTSA awards are intended to accelerate this type of research as well. It is worth noting that academic medical centers can often provide a unique advantage when conducting Phase 2 studies: access to patients with the disease of interest. This is particularly relevant for rare diseases, but also applies for more common conditions (see the chapter on "Orphan Drugs: Understanding the FDA Approval Process"). Since subject recruitment can be a major challenge for clinical trials, this can significantly enhance the ability to conduct Phase 2 (and 3) studies. Conflict-of-interest issues become increasingly common at this phase: in many cases, an academic entrepreneur who may profit financially from a compound is not allowed to directly recruit patients, especially from their own clinic, or perform many other research-related functions. 


\section{Phase 3 Clinical Research}

Phase 3 research consists of large, pivotal randomized trials that play a key role in establishing the clinical effectiveness of the compound to the FDA. Typically, there are two Phase 3 studies conducted for a compound, narrowly targeting the specific indication for which the pharmaceutical company hopes to obtain FDA approval. The sample size can vary, but is often greater than 500 research participants, and they are usually multisite studies due to the challenges associated with subject recruitment. These studies typically involve a placebo control, intent-to-treat analysis, and careful documentation of all adverse events. The findings are usually published in peer-reviewed journals by independent members of the academic community that led the research studies. Given the complexity and expense of Phase 3 studies, many startup companies may be acquired by a major pharmaceutical company after the Phase 2 research studies demonstrate efficacy and safety. This larger company often has the research experience and capacity to conduct the Phase 3 studies in a timely manner and move forward with the FDA process. Phase 3 clinical research studies typically require three years (“Drug Approvals - From Invention to Market...12 Years!”).

\section{New Drug Application}

Once the Phase 3 studies have concluded, the pharmaceutical company may elect to move forward with a New Drug Application (NDA) to the FDA. Preparing an NDA is a massive undertaking, with many NDAs consisting of over 100,000 pages of material. The NDA typically includes all the results of the research conducted to date, as well as information regarding planned marketing of the compound. As described by the FDA, this includes (Office of the Commissioner):

- Proposed labeling

- Safety updates

- Drug abuse information

- Patent information

- Any data from studies that may have been conducted outside the United States

- Institutional review board compliance information

- Directions for use

If all relevant components are provided, the FDA review team has 6-10 months to determine whether or not to approve the drug (Office of the Commissioner). As part of their review, the FDA will visit clinical sites that participated in the research to confirm that there was no evidence of fabrication of data, etc. In addition, the FDA may convene an independent advisory committee to obtain expert advice and public input on the compound and its planned indications for use.

\section{Phase 4 Research}

After approval by the FDA, the compound can be sold by the pharmaceutical company for the FDA-approved indications. However, the FDA may require additional research or data collection 
OVERVIEW OF DRUG DEVELOPMENT

to report on real-world outcomes related to the compound. These studies are referred to as "postmarketing studies."

\section{Conclusion}

Drug development is a highly regulated process that is largely defined by key FDA milestones that center around the investigational new drug application (IND) and the new drug application (NDA). Preclinical testing is crucial for IND approval, and the subsequent Phases 1-3 studies conducted under the IND help provide supporting evidence for the NDA. An academic entrepreneur can be closely involved in many of these steps, however, their institution's conflict-of-interest guidelines may place significant limits on the specific tasks they are allowed to perform at each step. Frequent engagement with the FDA and regulatory experts is crucial for the successful navigation of this complex process. Often, a startup may conduct the early phases of the drug development process, then sell the compound or be acquired by a larger pharmaceutical company for the later phases.

\section{Acknowledgments}

We would like to thank Dr. Tom Privette for his input on this chapter.

\section{Resources}

1. Drug Development Tools: The FDA provides a description of measures and methods that can facilitate the drug development process: https://www.fda.gov/drugs/developmentapproval-process-drugs/drug-development-tools-ddts.

2. The Center for Drug Evaluation and Research offers a series of helpful training courses and other events for small businesses: https://www.fda.gov/drugs/cder-small-businessindustry-assistance-sbia/cder-sbia-learn-webinars-conferences-trainings-upcomingevents.

\section{References}

DiMasi, Joseph A., et al. "Innovation in the Pharmaceutical Industry: New Estimates of R\&D Costs.” Journal of Health Economics, vol. 47, May 2016, pp. 20-33, doi:10.1016/j.jhealeco.2016.01.012.

“Drug Approvals-From Invention to Market...12 Years!” MedicineNet, https://www.medicinenet.com/script/main/art.asp?articlekey=9877. Accessed 29 Sept. 2019. Guidance for Industry and Review Staff Target Product Profile-A Strategic Development Process Tool. U.S. Food and Drug Administration, 9 Mar. 2007, https://www.fda.gov/media/72566/download.

Mullard, Asher. “2018 FDA Drug Approvals.” Nat. Rev. Drug Discov., vol. 18, no. 2, Feb. 2019, 
pp. 85-89, doi:10.1038/d41573-019-00014-X.

Office of the Commissioner. "The Drug Development Process." U.S. Food and Drug Administration, 2019, http://www.fda.gov/patients/learn-about-drug-and-deviceapprovals/drug-development-process.

Van Norman, Gail A. "Drugs, Devices, and the FDA: Part 1: An Overview of Approval Processes for Drugs.” JACC: Basic to Translational Science, vol. 1, no. 3, Apr. 2016, pp. 170-79, doi:10.1016/j.jacbts.2016.03.002.

Wikipedia contributors. "Drug Design.” Wikipedia, The Free Encyclopedia, 2 Sept. 2019, https://en.wikipedia.org/w/index.php?title=Drug_design\&oldid=913680280.

Wong, Chi Heem, et al. "Estimation of Clinical Trial Success Rates and Related Parameters."

Biostatistics, vol. 20, no. 2, Apr. 2019, pp. 273-86, doi:10.1093/biostatistics/kxx069.

\begin{abstract}
Chapter Last Updated 10/9/2019.
Please check Scholarly Commons (https://repository.upenn.edu/ace/) for the most recent version.

The contents of this chapter represent the opinions of the chapter authors and editors. The contents should not be construed as legal advice. The contents do not necessarily represent the official views of any affiliated organizations, partner organizations, or sponsors. For programs or organizations mentioned in this chapter, the authors encourage the reader to directly contact the relevant organization for additional information.
\end{abstract}

Content in this chapter is licensed by the editors under a Creative Commons AttributionNonCommercial-NoDerivatives 4.0 International (CC BY-NC-ND 4.0) license. 